\title{
Trombolisis intraarterial en trombosis de la arteria basilar. Recuperación de dos pacientes con síndrome de enclaustramiento
}

\author{
Patricio Mellado T, Patricio Sandoval R, José Tevah C, \\ Isidro H uete L, Luis Castillo F.

\section{Intra-arterial thrombolysis in locked-in syndrome. Report of two cases}

Locked-in syndrome is a dramatic clinical condition, the patient is awake, can listen and breath, but is unable to move any muscle, conserving only the vertical eye movements. The most common cause of locked-in syndrome is the thrombosis of the basilar artery and commonly leads to death, frequently due to pneumonia. Intravenous and intra arterial thrombolysis have been used successfully in a selective group of patients with ischemic stroke. There is only one report of two patients with locked-in syndrome who were treated successfully with intra arterial thrombolysis. Other authors, based in their experiences, do not recommend this treatment. We report two female patients aged 63 and 26 years, with Locked-in syndrome due to a basilar thrombosis who were treated successfully with intra arterial thrombolysis using ecombinant tissue plasminogen activator (r-TPA). The lapses between the onset of the symptoms and thrombolysis were 5 and 8 hours respectively. A complete recanalization was obtained in both patients during the thrombolysis. One year after, the first patient has only a moderate ataxia, walking with assistance and the other has a normal neurological examination (Rev Méd Chile 2004; 132: 357-60 ).

(Key Words: Locked-in syndrome; Plasminogen activators; Thrombolytic therapy; Vertebrobasilar insufficiency)

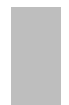

Recibido el 14 de julio, 2003. Aceptado en versión corregida el 5 de diciembre, 2003. Departamentos de Neurología, Radiología, Anestesiología y Programa de Medicina Intensiva. Facultad de Medicina, Hospital Clínico de la Pontificia Universidad Católica de Chile.

$\mathrm{E}^{1}$ síndrome de enclaustramiento corresponde a un cuadro clínico aterrador, se caracteriza por presentar tetraplejia y anartria, pero con preservación de la conciencia, audición, visión y respiración, es decir, el paciente se encuentra despierto pero es incapaz de comunicarse ni moverse. El signo que permite su diagnóstico es la preservación de los movimientos oculares verticales voluntarios, cuyo centro de integración se localiza en el mesencéfalo ${ }^{1}$. La etiopatogenia más frecuente es la isquemia de la protuberancia ${ }^{1}$. El pronóstico de la isquemia del troncoencéfalo es malo: 70 a $80 \%$ de los pacientes fallece o queda con graves secuelas

Correspondencia a: Dr. Patricio Mellado T. Marcoleta 347 Santiago, Chile. Fax: 56-2-632-6221. E-mail: pmellado@med.puc.cl neurológicas ${ }^{2}$, el pronóstico del síndrome de enclaustramiento de causa isquémico es ominoso, los pacientes habitualmente no mejoran su condición neurológica y la extensa mayoría fallece en pocos días debido a complicaciones médicas ${ }^{1,3}$.

Por otro lado, la trombolisis intravenosa e intraarterial es eficaz en el tratamiento de pacientes seleccionados con infarto encefálico, la ventana terapéutica debe ser de 3 y 6 h respectivamente ${ }^{4-7}$; la trombolisis intraarterial sólo ha sido evaluada con estudios bien diseñados en el teritorio carotíde $0^{5}$. Existen múltiples trabajos anecdóticos con trombolisis intraarterial en el temitorio vértebro-basilar-20, sus resultados son variables: se ha observado recanalización entre 40 y 78\%, buenos resultados clínicos entre 19 y 56\%, complicaciones hemorrágicas entre 0 y $17 \%$ y la mortalidad 
vańa entre 22 y $70 \%{ }^{21}$. Según nuestro conocimiento, la trombolisis intraarterial en pacientes con síndrome de enclaustramiento ha sido realizada con éxito sólo en $2 \operatorname{casos}^{12}$. En la literatura existe controversia sobre la indicación de la trombolisis intraarterial en estos pacientes, algunos autores no la recomiendan debido a la baja probabilidad de éxito ${ }^{14}$, otros en cambio, sugieren utilizar cualquier medida extraordinaria que pueda cambiar su ominosa historia natural ${ }^{22}$. A continuación describimos 2 pacientes con síndrome de enclaustramiento tratados con trombolisis intraarterial en nuestro hospital, en ambos casos se obtuvo una recanalización completa de la arteria basilar y una mejonía clínica significativa.

Paciente 1. Mujer de 63 años, hipertensa desde hacía 20 años, con mal control. La paciente despertó con disartria y hemiparesia izquierda, 12 $\mathrm{h}$ después y $2 \mathrm{~h}$ antes de su ingreso a nuestra institución, presentó compromiso de conciencia y tetraplejia. El examen general de ingreso era normal, estaba vigil, lo que podía evidenciarse sólo al solicitarle que movilizara los ojos en forma vertical, estaba anártrica, tetrapléjica y ventilaba espontáneamente. Se hizo el diagnóstico de síndrome de enclaustramiento. Se realizó una tomografía computada de encéfalo (TC) que mostró una hipodensidad en la base del puente con la arteria basilar espontáneamente hiperdensa. Se administró un bolo de heparina y a las $5 \mathrm{~h}$ de producido el síndrome se realizó una angiografía cerebral por sustracción digital; la familia otorgó el consentimiento para realizar una trombolisis intraarterial. La inyección de contraste a nivel del arco de la aorta mostró una obstrucción en el tercio medio de la arteria basilar (Figura 1). No fue posible realizar la inyección de activador de plasminógeno tisular (r-TPA) en forma local debido a la imposibilidad de entrar en las arterias vertebrales, la derecha era hipoplásica y la izquierda presentaba un asa que no fue posible franquear, se instiló r-TPA en el origen de la arteria vertebral izquierda, con $20 \mathrm{mg}$ de r-TPA se logró la lisis completa del trombo con visualización normal de la arteria basilar (Figura 2). Ocho horas después de la trombolisis la paciente recuperó el movimiento de la mirada y mejoró la hemiplejia derecha. El estudio de fuente embólica evidenció una fibrilación auricular paroxística, el resto del estudio fue normal. Se decidió anticoagular a la paciente, buscando un INR entre 2 y 3 . Al mes de evolución la paciente se encontraba con disartria,

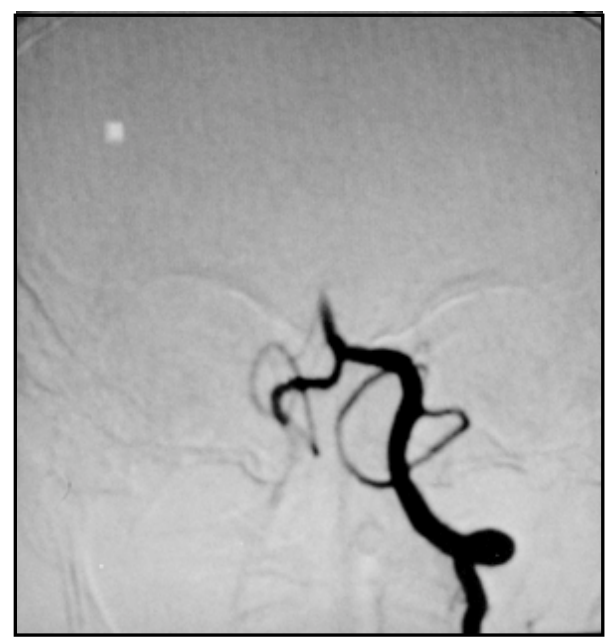

FIGURA 1.

disfagia leve, hemiplejia izquierda y risa intercalada con llanto espasmódico (parálisis pseudobulbar). Al año de evolución la paciente presentaba sólo ataxia, requiriendo de apoyo para la marcha.

Paciente 2. Mujer de 26 años con antecedente de migraña sin aura y usuaria de anticonceptivos orales. Estando previamente sana, presentó, al final del acto sexual, cefalea holocránea ictal de gran intensidad. $\mathrm{Al}$ ingreso a nuestra institución, $5 \mathrm{~h}$ después del inicio de la cefalea, presentaba un examen físico general normal y un examen neurológico con una tetraparesia severa, hemianestesia derecha, signo de Babinski bilateral, síndrome de Claude BernardHorner izquierdo, síndrome de uno y medio izquierdo y anartria; el movimiento vertical de los ojos y la conciencia estaba conservada. La TC de encéfalo de ingreso fue normal. Se diagnosticó un síndrome de enclaustramiento incompleto. Se inició tratamiento anticoagulante con heparina en bolo y a las tres horas de ingreso (ocho horas de evolución), se realizó una angiografía por sustracción digital que confirmó la oclusión de la arteria basilar en su tercio medio y descartó una disección. Después de obtener el consentimiento de la familia, se realizó una trombolisis intraarterial y local, de tipo mecánica y química con $40 \mathrm{mg}$ de r-TPA, se obtuvo una repermeabilización completa de la arteria basilar. En algunos minutos se observó mejonía de la hemiplejia derecha, sin embargo, a las tres horas postangiografía presentó compromiso de conciencia con rigidez de descerebración. La TC de control mostró un hemorragia mesencefálica petequial. Se revirtió la 


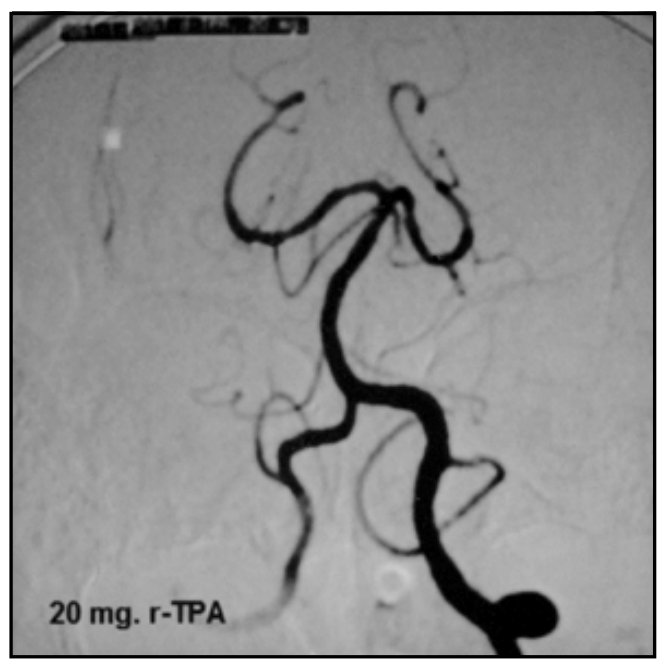

FIGURA 2.

anticoagulación con protamina. Al segundo día mejoró la conciencia y posteriormente, con neurorehabilitación intensiva comenzó a mejorar el resto de los déficits neurológicos. Una resonancia magnética de encéfalo, al mes de evolución, mostró un infarto de la arteria cerebelosa superior izquierda. El estudio de fuente embólica reveló una malformación trabecular de la aurícula derecha y un foramen oval de $10 \mathrm{~mm}$. El resto del estudio fue normal (doppler carotídeo-vertebral, trombofilias, estudio neuroinmunológico, angiografía de arco de la aorta y vasos de cuello y doppler de extremidades inferiores). Se reinició la anticoagulación a la semana de suspendida, buscando un INR entre 2 y 3 . A los ocho meses de evolución sólo presentaba dismetría izquierda leve. Un año después, su examen neurológico era normal y había retomado sus actividades habituales.

\section{DisCUSIÓN}

Describimos dos pacientes con un síndrome de enclaustramiento, en la primera la TC de encéfalo mostraba a la arteria basilar hiperdensa con hipodensidad en la base de la protuberancia, en la segunda la TC fue normal; la angiografía con substracción digital evidenció una obstrucción en el tercio medio de la arteria basilar en ambos casos. El estudio de fuente embólica mostró una fibrilación auricular paroxística y un foramen oval permeable respectivamente. Ambas pacientes llegaron a las $2 \mathrm{~h}$ de haber presentado el síndrome de enclaustramiento, sin embargo, la primera había despertado $12 \mathrm{~h}$ antes con hemiparesia izquierda y disartria, se insistió en explicar a la familia que buscańamos mejorar el déficit neurológico actual pero que creíamos, debido al tiempo transcumido, que el déficit neurológico con el cual había despertado no mejorańa. Ambas familias otorgaron su consentimiento. Ambas pacientes fueron anticoaguladas con bolo de 5.000 unidades de heparina seguidas de una infusión continua y sometidas a una angiografía diagnóstica con sustracción digital a las 5 y $8 \mathrm{~h}$ de producido el síndrome. En ambas pacientes se logró la repermeabilización completa de la arteria basilar durante el procedimiento con r-TPA en dosis de 20 y $40 \mathrm{mg}$ respectivamente. La primera paciente presentó una lenta y progresiva mejońa, al año de evolución presentaba sólo ataxia de la marcha, requiniendo asistencia para deambular. La segunda paciente presentó como complicación una transformación hemomágica petequial en el mesencéfalo, sin embargo, comenzó a mejorar clínicamente a partir del primer mes y al año se encontraba asintomática y realizando una vida normal.

La trombolisis intravenosa e intraarterial han sido eficaces en grupos seleccionados de pacientes ${ }^{4-7}$. Respecto a la trombolisis intraarterial en el teritorio vértebro-basilar sólo existen reportes anecdóticos ${ }^{8-20}$. En la actualidad hay controversia respecto a si se debiera ofrecer trombolisis intraarterial a pacientes con un síndrome de enclaustramiento ${ }^{12,14}$. Según nuestro conocimiento, sólo se han publicado 2 casos de trombolisis intraarterial en estos pacientes con buenos resultados ${ }^{12}$. Nuestra opinión es que frente a pacientes con este síndrome, con una ventana terapéutica de hasta $12 \mathrm{~h}$ y que no presenten contraindicación absoluta para la trombolisis intraarterial, ésta debe ser intentada por un equipo multidisciplinario, seguido de una terapia de neuromehabilitación intensiva y siempre se debe contar con el consentimiento informado de la familia 0 , si es posible, del paciente.

\section{CONCLUSIÓN}

El síndrome de enclaustramiento es un cuadro clínico aterrador y con un pronóstico ominoso, cuando su causa es la isquemia de la protuberancia la trombolisis intraarterial puede ser utilizada. Un trabajo previo describe 2 casos con resultados satisfactorios. Nuestra experiencia con 2 casos mostró que la trombolisis intraarterial en pacientes con un síndrome de enclaustramiento puede ser eficaz en revertir esta condición, evitando el fallecimiento y logrando una excelente recuperación neurológica.

Es necesario un trabajo bien diseñado que compare la trombolisis intraarterial y la heparina 
en el territorio vértebro-basilar para determinar su real eficacia. Hasta que se realicen estos estudios, nuestro grupo sugiere realizar como una terapia

\section{REFERENCIAS}

1. Sandoval P, Meliado P. Síndrome de Locked-In. Cuad Neurol 2000; 24: 102-11.

2. Patterson J, Grabois M. Locked-in syndrome: A review of 139 cases. Stroke 1986; 17: 758-64.

3. Caplan L Basilar artery occlusive disease. En: Caplan L, ed. Posterior Circulation Diseases. Clinical, findings, diagnosis and management. Cambridge. Blackwell Science 1996; 324-80.

4. Tissue plasminogen activator for acute ischemic stroke. The National Institute of Neurological Disorders and Stroke r-TPA Stroke Study Group. N Engl J Med 1995; 333: 1581-7.

5. Furlan A, Higashida R, Wechsier L, Gent M, Rowiey $\mathrm{H}, \mathrm{KASE} \mathrm{C}$ ET AL. For the PROACT investigators. Intraarterial prourokinase for acute ischemic stroke. The PROACT II study: A randomized controlled trial. JAMA 1999; 282: 2003-11.

6. Del Zoppo GJ, Hamann G, Hosomi N. Thrombolytic therapy. En: Fisher M, ed. Stroke Therapy. Boston. Butterworth-Heinemann. 2001; 261-74.

7. Furlan AJ, Higashida R, Katzan I, Abou-Chebl A. Intraarterial thrombolysis in acute ischemic stroke. En: Lyden PD, ed. Thrombolytic therapy for stroke. Totowa. Humana Press 2001; 175-95.

8. Cross DT, Moran CJ, Akins PT, Angtuaco EE, DIRINGER MN. Relationship between clot location and outcome after basilar artery thrombolysis. Am J Neuroradiol 1997; 18: 1221-8.

9. BeCKer KJ, Monsein LH, Ulatowski J, Mirski M, Wimams M, HANIEY DF. Intraarterial thrombolysis in vertebrobasilar occlusion. Am J Neuroradiol 1996; 17: 255-62.

10. Brandt T, Von Kummer R, Mu山er-Kuppers M, Hacke W. Thrombolytic therapy of acute basilar artery occlusion. Variables affecting recanalization and outcome. Stroke 1996; 27: 875-81.

11. Zeumer H, Freitag HJ, Grzyska U, Neunzig HP. Local intraarterial fibrinolysis in acute vertebrobasilar occlusion. Technical developments and recent results. Neuroradiology 1989; 31: 336-40.

12. Wijdicks EFM, Nichols D, Thielen K, Fulgham J, BROWN R, MEISSNER I ET AL. Intraarterial thrombolysis in acute basilar artery thromboembolism: The de rescate, una trombolisis intraarterial en pacientes seleccionados que sufran un síndrome de enclaustramiento debido a isquemia.

initial Mayo Clinic experience. Mayo Clin Proc 1997; 72: 1005-13.

13. Milhaud D, Charif M, Héroum $C$, Smadja $P$, Bonafé A, Pages M et al. Thrombolyse par voie artérielle et angioplastie dans l'occlusion athéromateuse du tronc basilaire. Rev neurol 2001; 157: 423-6.

14. Hacke W, Zeumer H, Ferbert A, Brückmann H, Del Zoppo GJ. Intraarterial thrombolytic therapy improves outcome in patients with acute vertebrobasilar occlusive disease. Stroke 1988; 19: 1216-22.

15. Levy EL, FiruK AD, Wisniewski S, Rubin G, Jungreis CAA, WECHSLER LR ET AL. Factors affecting survival rates for acute vertebrobasilar artery occlusions treated with intraarterial thrombolytic therapy: A metaanalytical approach. Neurosurgery 1999; 45: 539-48.

16. Solaz J, Martínez-Rodrigo J, Lonjedo E, Poyatos C, Vega M, Palmeo J. Fibrinolisis intrarterial en la trombosis aguda de la arteria basilar. Rev Neurol 1998; 27: 1012-4.

17. Becker KJ, Purcell LL, Hacke W, Hanley DF. Vertebrobasilar thrombosis: Diagnosis management and the use of intra-arterial thrombolytic. Crit Care Med 1996; 24: 1729-42.

18. Phatouros CC, Higashida RD, Malek AM, Smith WS, MuLy TW, DeARmond SJ et AL. Endovascular stenting of an acute thrombosed basilar artery: Technical case report and review of the literature. Neurosurgery 1999; 44: 667-73.

19. Nakayama T, TanaKa $K$, KaneKo $M$, Yokoyama $T$, UEmURA K. Thrombolysis and angioplasty for acute occlusion of intracranial vertebrobasilar arteries. Report of three cases. J Neurosurg 1998; 88: 919-22.

20. Barr JD, Mathis JM, WiLdenhain SL, Wechsier L, Jungreis CA, HorTon JA. Acute stroke intervention with intraarterial urokinase infusion. J Vasc Interv Radiol 1994; 5: 705-13.

21. PHAN T, WIJDICKS EFM. Intraarterial thrombolysis for vertebrobasilar circulation ischemia. Crit Care Clin 1999; 15: 719-42.

22. WiJDICKS EFM. Acute basilar artery occlusion. En: Wijdicks EFM, ed. The clinical practice of critical care neurology. Philadelphia. Lippincott-Raven 2003; 291-305. 